\author{
M.T. Jenaliyev ${ }^{1}$, S.A. Iskakov ${ }^{2}$, M.I. Ramazanov ${ }^{2,3}$, Zh.M. Tuleutaeva² \\ ${ }^{1}$ Institute of Mathematics and Mathematical Modeling CS MES RK, Almaty, Kazakhstan; \\ ${ }^{2}$ Ye.A. Buketov Karaganda State University, Kazakhstan; \\ ${ }^{3}$ Institute of Applied Mathematics, Karaganda, Kazakhstan \\ (E-mail: erasl-79@mail.ru)
}

\title{
On the solvability of the first boundary value problem for the loaded equation of heat conduction
}

\begin{abstract}
In this paper we consider the first boundary value problem for the loaded equation of heat conduction in a quarter plane. The loaded term is the trace of the fractional derivative of order $\nu, \quad 0 \leq \nu \leq 1$ with respect to the time variable on the line $x=t$. It is shown that when $0 \leq \nu \leq 1$ and $\forall \lambda \in \mathrm{C}$, then the load is a weak perturbation, that is, the studied problem has a unique solution in the class of bounded functions.
\end{abstract}

Keywords: loaded equation of heat conduction, boundary value problem, fractional derivative, Volterra integral equation.

Statement of the problem. In the domain $Q=\left\{x \in R_{+}, t \in R_{+}\right\}$the following boundary value problem is considered:

$$
\begin{gathered}
\frac{\partial u}{\partial t}-a^{2} \frac{\partial^{2} u}{\partial x^{2}}+\left.\lambda \cdot{ }_{0} D_{t}^{\nu} u(x, t)\right|_{x=t}=f(x, t) \\
u(x, 0)=0, \quad u(0, t)=0
\end{gathered}
$$

where

$$
\lambda \in C, 0 \leq \nu<1,{ }_{0} D_{t}^{\nu} u(x, t)=\frac{1}{\Gamma(1-\nu)} \frac{\partial}{\partial t} \int_{0}^{t} \frac{1}{(t-\tau)^{\nu}} u(x, \tau) d \tau-
$$

is the fractional Riemann-Liouville derivative of the function $u(x, t)$ with respect to a variable $t$ of order $\nu$,

$$
{ }_{0} D_{t}^{\nu}\left\{\int_{0}^{t} \int_{0}^{\infty} G(x, \xi, t-\tau) \cdot f(\xi, \tau) d \xi d \tau\right\} \in M\left(R_{+}\right) .
$$

Hear

$$
\begin{gathered}
M\left(R_{+}\right)=L_{\infty}\left(R_{+}\right) \cap C\left(R_{+}\right) \\
G(x, \xi, t)=\frac{1}{2 \sqrt{\pi t}}\left\{\exp \left(-\frac{(x-\xi)^{2}}{4 t}\right)-\exp \left(-\frac{(x+\xi)^{2}}{4 t}\right)\right\}-
\end{gathered}
$$

is the Green's function of the first boundary value problem for the heat equation.

Peculiarity of this problem lies in the fact that the loaded term is the value of the fractional derivative on the line $\mathrm{x}=\mathrm{t}$. Similar problems were considered in [1, 2]. In [1] it has been shown that when $\nu=1$ the load is a strong perturbation and for certain values of the parameter $\lambda$ the corresponding homogeneous problem has nonzero solutions, that is, a non-homogeneous problem has a non-unique solution.

The purpose of this paper is to determine the nature of the load when $0 \leq \nu<1$.

Inverting the differential part in the boundary value problem (1)-(2), we have the following representation for the solution:

$$
\begin{aligned}
u(x, t)= & -\left.\lambda \int_{0}^{t} \int_{0}^{\infty} G(x, \xi, t-\tau) \cdot\left[{ }_{0} D_{t}^{\nu} u(\xi, \tau)\right]\right|_{\xi=\tau} d \xi d \tau+ \\
& +\int_{0}^{t} \int_{0}^{\infty} G(x, \xi, t-\tau) \cdot f(\xi, \tau) d \xi d \tau,
\end{aligned}
$$


or

$$
\begin{aligned}
u(x, t)=-\lambda & \left.\int_{0}^{t} \int_{0}^{\infty} K_{0}(x, t-\tau) \cdot\left[{ }_{0} D_{t}^{\nu} u(\xi, \tau)\right]\right|_{\xi=\tau} d \xi d \tau+ \\
& +\int_{0}^{t} \int_{0}^{\infty} G(x, \xi, t-\tau) \cdot f(\xi, \tau) d \xi d \tau,
\end{aligned}
$$

hear

$$
K_{0}(x, t-\tau)=\int_{0}^{\infty} G(x, \xi, t-\tau) d \xi=\operatorname{erf}\left(\frac{x}{2 \sqrt{t-\tau}}\right)
$$

Indeed

$$
\begin{aligned}
\int_{0}^{\infty} G(x, \xi, t-\tau) d \xi= & \frac{1}{2 \sqrt{\pi(t-\tau)}} \int_{0}^{\infty}\left\{\exp \left(-\frac{(x-\xi)^{2}}{4(t-\tau)}\right)-\exp \left(-\frac{(x+\xi)^{2}}{4(t-\tau)}\right)\right\} d \xi= \\
& =\left\|\eta \frac{x \mp \xi}{2 \sqrt{t-\tau}}, \quad z=\frac{x}{2 \sqrt{t-\tau}}\right\|= \\
= & \frac{1}{\sqrt{\pi}}\left\{\int_{-\infty}^{z} \exp \left(-\eta^{2}\right) d \eta-\int_{z}^{\infty} \exp \left(-\eta^{2}\right) d \eta\right\}= \\
= & \frac{2}{\sqrt{\pi}} \int_{0}^{z} \exp \left(-\eta^{2}\right) d \eta=\operatorname{erf}\left(\frac{x}{2 \sqrt{t-\tau}}\right) .
\end{aligned}
$$

Substituting the value of this integral into (4), we obtain

$$
u(x, t)=-\left.\lambda \int_{0}^{t} \operatorname{erf}\left(\frac{x}{2 \sqrt{t-\tau}}\right) \cdot\left[{ }_{0} D_{t}^{\nu} u(\xi, \tau)\right]\right|_{\xi=\tau} d \tau+f_{1}(x, t)
$$

where

$$
f_{1}(x, t)=\int_{0}^{t} \int_{0}^{\infty} G(x, \xi, t-\tau) \cdot f(\xi, \tau) d \xi d \tau
$$

It follows from (5) that in order to find solutions of problem (1)-(2) it is sufficient find an expression $\left.\left[{ }_{0} D_{t}^{\nu} u(\xi, \tau)\right]\right|_{\xi=\tau}$.

For this, we differentiate both sides of (5) by $t$, then assuming $x=t$, we get

$$
\begin{gathered}
{\left.\left[{ }_{0} D_{t}^{\nu} u(x, t)\right]\right|_{x=t}=-\left.\lambda\left\{\left.{ }_{0} D_{t}^{\nu} \int_{0}^{t} \operatorname{erf}\left(\frac{x}{2 \sqrt{t-\tau}}\right) \cdot\left[{ }_{0} D_{t}^{\nu} u(\xi, \tau)\right]\right|_{\xi=\tau} d \tau\right\}\right|_{x=t}+} \\
+\left.{ }_{0} D_{t}^{\nu} f_{1}(x, t)\right|_{x=t},
\end{gathered}
$$

and introducing the notation

$$
\left.\left[{ }_{0} D_{t}^{\nu} u(x, t)\right]\right|_{x=t}=\varphi(t),\left.{ }_{0} D_{t}^{\nu} f_{1}(x, t)\right|_{x=t}=f_{2}(t) ;
$$

from (6) we obtain the following integral equation

$$
\varphi(t)+\left.\lambda\left\{{ }_{0} D_{t}^{\nu} \int_{0}^{t} \operatorname{erf}\left(\frac{x}{2 \sqrt{t-\tau}}\right) \cdot \varphi(\tau) d \tau\right\}\right|_{x=t}=f_{2}(t) .
$$


Next, we calculate

$$
\begin{gathered}
{ }_{0} D_{t}^{\nu} \int_{0}^{t} \operatorname{erf}\left(\frac{x}{2 \sqrt{t-\tau}}\right) \cdot \varphi(\tau) d \tau= \\
=\frac{1}{\Gamma(1-\nu)} \frac{\partial}{\partial t} \int_{0}^{t} \frac{1}{(t-\tau)^{\nu}}\left\{\int_{0}^{\tau} \operatorname{erf}\left(\frac{x}{2 \sqrt{\tau-\tau_{1}}}\right) \cdot \varphi\left(\tau_{1}\right) d \tau_{1}\right\} d \tau= \\
=\frac{1}{\Gamma(1-\nu)} \frac{\partial}{\partial t} \int_{0}^{t}\left\{\int_{\tau_{1}}^{\tau} \frac{1}{(t-\tau)^{\nu}} \cdot \operatorname{erf}\left(\frac{x}{2 a \sqrt{\tau-\tau_{1}}}\right) d \tau\right\} \varphi\left(\tau_{1}\right) d \tau_{1}=\left\|\begin{array}{l}
t-\tau=\eta \\
\tau=t-\eta
\end{array}\right\|= \\
=\frac{1}{\Gamma(1-\nu)} \int_{0}^{t} \frac{\partial}{\partial t}\left\{\int_{0}^{t-\tau_{1}} \frac{1}{\eta^{\nu}} \cdot \operatorname{erf}\left(\frac{x}{2 a \sqrt{t-\tau_{1}-\eta}}\right) d \eta \varphi\left(\tau_{1}\right) d \tau_{1}=\right. \\
\quad \int_{0}^{t}\left\{\frac{1}{\left(t-\tau_{1}\right)^{v}} \cdot \operatorname{erf}(\infty)-\int_{0}^{t} \frac{1}{\eta^{\nu}} \cdot \frac{2}{\sqrt{\pi}} \cdot \exp \left\{-\frac{x^{2}}{4 a^{2}\left(t-\tau_{1}-\eta\right)}\right\} \times\right. \\
\left.\times \frac{x}{4 a\left(t-\tau_{1}-\eta\right)^{\frac{3}{2}}} d \eta\right\} \varphi\left(\tau_{1}\right) d \tau_{1}=\frac{1}{\Gamma(1-\nu)} \int_{0}^{t} K_{\nu}\left(t, \tau_{1}, x\right) \varphi\left(\tau_{1}\right) d \tau_{1},
\end{gathered}
$$

where the following notation is used:

$$
\begin{gathered}
K_{\nu}(t, \tau, x)=\frac{1}{\left(t-\tau_{1}\right)^{v}}-k_{\nu}\left(t, \tau_{1}, x\right) \\
k_{\nu}\left(t, \tau_{1}, x\right)=\frac{x}{2 a \sqrt{\pi}} \int_{0}^{t-\tau_{1}} \frac{1}{\eta^{\nu}} \cdot \frac{1}{\left(t-\tau_{1}-\eta\right)^{\frac{3}{2}}} \cdot \exp \left\{-\frac{x^{2}}{4 a^{2}\left(t-\tau_{1}-\eta\right)}\right\} d \eta .
\end{gathered}
$$

Let us find the explicit form of the function $k_{\nu}\left(t, \tau_{1}, x\right)$.

$$
\begin{aligned}
& k_{\nu}\left(t, \tau_{1}, x\right)=\frac{x}{2 a \sqrt{\pi}} \int_{0}^{t-\tau_{1}} \frac{1}{\eta^{\nu}} \cdot \frac{1}{\left(t-\tau_{1}-\eta\right)^{\frac{3}{2}}} \cdot \exp \left\{-\frac{x^{2}}{4 a^{2}\left(t-\tau_{1}-\eta\right)}\right\} d \eta= \\
& =\left\|\begin{array}{l}
\eta=\left(t-\tau_{1}\right)(1-z) \\
d \eta=-\left(t-\tau_{1}\right) d z
\end{array}\right\|= \\
& =\frac{x}{2 a \sqrt{\pi}} \int_{0}^{1} \frac{t-\tau_{1}}{\left(t-\tau_{1}\right)^{\nu}(1-z)^{\nu}} \cdot \frac{1}{\left(t-\tau_{1}\right)^{\frac{3}{2}} \cdot z^{\frac{3}{2}}} \cdot \exp \left\{-\frac{x^{2}}{4 a^{2}\left(t-\tau_{1}\right)} \cdot \frac{1}{z}\right\} d z= \\
& =\frac{x}{2 a \sqrt{\pi}} \cdot \frac{1}{\left(t-\tau_{1}\right)^{\nu+\frac{1}{2}}} \int_{0}^{1} z^{-\frac{3}{2}} \cdot(1-z)^{-\nu} \cdot \exp \left\{-\frac{x^{2}}{4 a^{2}\left(t-\tau_{1}\right)} \cdot \frac{1}{z}\right\} d z= \\
& =\|[3] \cdot p .367, \quad 3.471(2)\|= \\
& =\Gamma(1-\nu) \cdot \frac{x}{2 a \sqrt{\pi}} \cdot \frac{1}{\left(t-\tau_{1}\right)^{\nu+\frac{1}{2}}} \cdot\left[\frac{x^{2}}{4 a^{2}\left(t-\tau_{1}\right)}\right]^{-\frac{3}{4}} \cdot \exp \left\{-\frac{x^{2}}{8 a^{2}\left(t-\tau_{1}\right)}\right\} \times \\
& \times W_{\nu-\frac{1}{4},-\frac{1}{4}}\left\{\frac{x^{2}}{4 a^{2}\left(t-\tau_{1}\right)}\right\}= \\
& =\Gamma(1-\nu) \cdot \frac{\sqrt{2 a}}{\sqrt{\pi x}} \cdot \frac{1}{\left(t-\tau_{1}\right)^{\nu-\frac{1}{4}}} \cdot \exp \left\{-\frac{x^{2}}{8 a^{2}\left(t-\tau_{1}\right)}\right\} \cdot W_{\nu-\frac{1}{4},-\frac{1}{4}}\left\{\frac{x^{2}}{4 a^{2}\left(t-\tau_{1}\right)}\right\},
\end{aligned}
$$

hear $W_{\nu-\frac{1}{4},-\frac{1}{4}}\left\{\frac{x^{2}}{4 a^{2}\left(t-\tau_{1}\right)}\right\}$ is Whittaker function. 
Substituting the function $k_{\nu}\left(t, \tau_{1}, x\right)$ into $(9)$, for the kernel $K_{\nu}(t, \tau, x)$ finally we obtain the following representation

$$
\begin{gathered}
K_{\nu}(t, \tau, x)=\frac{1}{\Gamma(1-\nu) \cdot\left(t-\tau_{1}\right)^{\nu}}-\sqrt{\frac{2 a}{\pi}} \cdot \frac{1}{\sqrt{x}} \times \\
\times \frac{1}{\left(t-\tau_{1}\right)^{\nu-\frac{1}{4}}} \exp \left\{-\frac{x^{2}}{8 a^{2}\left(t-\tau_{1}\right)}\right\} \cdot W_{\nu-\frac{1}{4},-\frac{1}{4}}\left\{\frac{x^{2}}{4 a^{2}\left(t-\tau_{1}\right)}\right\} .
\end{gathered}
$$

Thus, the integral equation (7) takes the form

$$
\varphi(t)-\lambda \int_{0}^{t} K_{\nu}(t, \tau) \cdot \varphi(\tau) d \tau=f_{2}(t)
$$

where

$$
K_{\nu}(t, \tau)=\sqrt{\frac{2 a}{\pi}} \cdot \frac{1}{\sqrt{t} \cdot(t-\tau)^{\nu-\frac{1}{4}}} \exp \left\{-\frac{t^{2}}{8 a^{2}(t-\tau)}\right\} \cdot W_{\nu-\frac{1}{4},-\frac{1}{4}}\left\{\frac{t^{2}}{4 a^{2}(t-\tau)}\right\}-\frac{1}{\Gamma(1-\nu) \cdot(t-\tau)^{\nu}} .
$$

This kernel has a weak singularity when $0 \leq \nu<1$, that will be shown below.

We consider some particular cases of equation (11) for specific values of $\nu: \nu=1, \nu=0, \nu=\frac{1}{2}$.

We note that earlier in [1] the problem (1)-(2) was considered for the case $\nu=1$, which was reduced to an integral equation of the form

$$
(1+\lambda) \varphi(t)-\lambda \int_{0}^{t} K_{1}(t, \tau) \cdot \varphi(\tau) d \tau=f_{2}(t)
$$

the kernel of this integral equation

$$
K_{1}(t, \tau)=\frac{1}{2 a \sqrt{\pi}} \cdot \frac{t}{(t-\tau)^{\frac{3}{2}}} \cdot \exp \left\{-\frac{t^{2}}{4 a^{2}(t-\tau)}\right\}-
$$

is «incompressible», since

$$
\lim _{t \rightarrow 0} \int_{0}^{t} K_{1}(t, \tau) d \tau=1
$$

and it was shown that the corresponding homogeneous integral equation has non-zero solutions, and their number depends on $|\lambda|$.

We show that when $\nu \rightarrow 1-0$, the integral equation (11) coincides with equation (12). To do this in (11) we pass to the limit $\nu \rightarrow 1-0$, that is, we calculate the following limits:

$$
\begin{gathered}
\lim _{\nu \rightarrow 1-0} \lambda \cdot \int_{0}^{t} \frac{1}{\Gamma(1-\nu) \cdot(t-\tau)^{\nu}} \cdot \varphi(\tau) d \tau= \\
=\left\|\begin{array}{l}
u=\varphi(\tau) ; d u=\varphi^{\prime}(\tau) d \tau \\
d v=\frac{d \tau}{(t-\tau)^{\nu}} ; v=-\frac{1}{1-\nu}(t-\tau)^{1-\nu}
\end{array}\right\|= \\
=\lim _{\nu \rightarrow 1-0} \frac{1}{\Gamma(1-\nu)} \cdot \lambda\left\{-\left.\frac{(t-\tau)^{1-\nu}}{1-\nu} \cdot \varphi(\tau)\right|_{0} ^{t}+\frac{1}{1-\nu} \int_{0}^{t}(t-\tau)^{1-\nu} \cdot \varphi^{\prime}(\tau) d \tau\right\}= \\
=\lim _{\nu \rightarrow 1-0} \frac{\lambda}{(1-\nu) \cdot \Gamma(1-\nu)} \cdot\left\{t^{1-\nu} \cdot \varphi(0)+\int_{0}^{t}(t-\tau)^{1-\nu} \cdot \varphi^{\prime}(\tau) d \tau\right\}= \\
=\lambda \cdot \varphi(0)+\left.\lambda \cdot \varphi(\tau)\right|_{0} ^{t}=\lambda \cdot \varphi(0)+\lambda \cdot \varphi(t)-\lambda \cdot \varphi(0)=\lambda \cdot \varphi(t) .
\end{gathered}
$$


Now we find the limit from the first summand

$$
\begin{gathered}
\lim _{\nu \rightarrow 1-0} k_{\nu}(t, \tau)=\sqrt{\frac{2 a}{\pi}} \cdot \frac{1}{\sqrt{t} \cdot(t-\tau)^{\frac{3}{4}-0}} \times \\
\times \exp \left\{-\frac{t^{2}}{8 a^{2}(t-\tau)}\right\} \cdot W_{\frac{3}{4}-0,-\frac{1}{4}}\left\{\frac{t^{2}}{4 a^{2}(t-\tau)}\right\}= \\
=\sqrt{\frac{2 a}{\pi}} \cdot \frac{1}{\sqrt{t} \cdot(t-\tau)^{\frac{3}{4}-0}} \cdot \exp \left\{-\frac{t^{2}}{8 a^{2}(t-\tau)}\right\} \times \\
\times\left\{\frac{\Gamma\left(\frac{1}{2}\right)}{\Gamma(+0)} \cdot M_{\frac{3}{4},-\frac{1}{4}}\left[\frac{t^{2}}{4 a^{2}(t-\tau)}\right]+\frac{\Gamma\left(-\frac{1}{2}\right)}{\Gamma\left(-\frac{1}{2}\right)} \cdot M_{\frac{3}{4},-\frac{1}{4}}\left[\frac{t^{2}}{4 a^{2}(t-\tau)}\right]\right\} .
\end{gathered}
$$

Here we use the representation of the Whittaker function $W_{\lambda, \mu}(z)$ in terms of the function $M_{\lambda, \mu}(z)$ $[3 ; 1024(9.220)]$. Further, writing down the Whittaker function $M_{\lambda, \mu}(z)$ via the Kummer function $(\alpha, \beta ; z)$ we have:

$$
\begin{aligned}
\lim _{\nu \rightarrow 1-0} k_{\nu}(t, \tau)= & \sqrt{\frac{2 a}{\pi}} \cdot \frac{1}{\sqrt{t} \cdot(t-\tau)^{\frac{3}{4}-0}} \cdot \exp \left\{-\frac{t^{2}}{8 a^{2}(t-\tau)}\right\} \cdot\left[\frac{t^{2}}{4 a^{2}(t-\tau)}\right]^{\frac{3}{4}} \times \\
& \times \exp \left\{-\frac{t^{2}}{8 a^{2}(t-\tau)}\right\} \cdot \Phi\left(0, \frac{3}{2}, \frac{t^{2}}{4 a^{2}(t-\tau)}\right)= \\
& =\frac{1}{2 a \sqrt{\pi}} \cdot \frac{t}{(t-\tau)^{\frac{3}{2}}} \cdot \exp \left\{-\frac{t^{2}}{4 a^{2}(t-\tau)}\right\}
\end{aligned}
$$

Thus, we obtain

$$
(1+\lambda) \varphi(t)-\lambda \int_{0}^{t} \frac{1}{2 a \sqrt{\pi}} \cdot \frac{t}{(t-\tau)^{\frac{3}{2}}} \cdot \exp \left\{-\frac{t^{2}}{4 a^{2}(t-\tau)}\right\} \cdot \varphi(\tau) d \tau=f_{2}(t) .
$$

Indeed, equation (13) coincides with equation (12) [1].We further define the form of the integral equation (11) at $\nu \rightarrow 0$,

For this, we find the value $K_{\nu}(t, \tau)$ при $\nu \rightarrow 0$.

$$
\begin{gathered}
\lim _{\nu \rightarrow 0} K_{\nu}(t, \tau)=1-\sqrt{\frac{2 a}{\pi}} \cdot \frac{(t-\tau)^{\frac{1}{4}}}{\sqrt{t}} \cdot \exp \left\{-\frac{t^{2}}{8 a^{2}(t-\tau)}\right\} \cdot W_{-\frac{1}{4},-\frac{1}{4}}\left\{\frac{t^{2}}{4 a^{2}(t-\tau)}\right\}= \\
=\left\|W_{-\frac{1}{4},-\frac{1}{4}}(z)=[1-\operatorname{erf}(x)] \cdot \frac{\sqrt{\pi} \cdot \sqrt{x}}{e^{-\frac{x^{2}}{2}}}\right\|= \\
=1-\sqrt{\frac{2 a}{\pi}} \cdot \frac{(t-\tau)^{\frac{1}{4}}}{\sqrt{t}} \cdot \frac{\sqrt{\pi} \cdot \sqrt{t}}{(2 a)^{\frac{1}{2}} \cdot(t-\tau)^{\frac{1}{4}}} \cdot\left[1-\operatorname{erf}\left\{\frac{t}{2 a \sqrt{t-\tau}}\right\}\right]= \\
=1-\left[1-\operatorname{erf}\left\{\frac{t}{2 a \sqrt{t-\tau}}\right\}\right]=\operatorname{erf}\left\{\frac{t}{2 a \sqrt{t-\tau}}\right\} .
\end{gathered}
$$

Using this relation, we obtain the following integral equation

$$
\varphi(t)-\lambda \int_{0}^{t} \operatorname{erf}\left\{\frac{t}{2 a \sqrt{t-\tau}}\right\} \cdot \varphi(\tau) d \tau=f_{2}(t)
$$

where $\varphi(t)=\left.u(x, t)\right|_{x=t}$.

This equation coincides with the integral equation (5), when $\nu \rightarrow 0$. 
Now we consider the case $\nu \rightarrow \frac{1}{2}$. That is

$$
\begin{gathered}
\lim _{\nu \rightarrow \frac{1}{2}} K_{\nu}(t, \tau)=\sqrt{\frac{2 a}{\pi}} \cdot \frac{1}{\sqrt{t} \cdot(t-\tau)^{\frac{1}{4}}} \exp \left\{-\frac{t^{2}}{8 a^{2}(t-\tau)}\right\} \cdot W_{\frac{1}{4},-\frac{1}{4}}\left\{\frac{t^{2}}{4 a^{2}(t-\tau)}\right\}- \\
-\frac{1}{\sqrt{\pi}} \cdot \frac{1}{\sqrt{t-\tau}} .
\end{gathered}
$$

To determine the specific form of the kernel, we use the representation of the Whittaker function

$$
\begin{aligned}
& W_{\frac{1}{4},-\frac{1}{4}}\{z\}=M_{\frac{1}{4},-\frac{1}{4}}(z)+\frac{\Gamma\left(-\frac{1}{2}\right)}{\Gamma(+0)} \cdot M_{\frac{1}{4}, \frac{1}{4}}(z)= \\
& =z^{\frac{1}{4}} \cdot e^{-\frac{z}{2}}+\frac{\Gamma\left(-\frac{1}{2}\right)}{\Gamma(+0)} \cdot \frac{\sqrt{\pi}}{2} \cdot z^{\frac{1}{4}} \cdot e^{-\frac{z}{2}} \cdot \operatorname{erfi}\{\sqrt{z}\},
\end{aligned}
$$

where $\operatorname{erfi}\{\sqrt{z}\}=\int_{0}^{\sqrt{z}} e^{\xi^{2}} d \xi$. Hence the kernel $K_{\frac{1}{2}}(t, \tau)$ is equal to the sum of three terms.

We compute the first summand

$$
\begin{gathered}
K_{\frac{1}{2}}^{(1)}(t, \tau)=\sqrt{\frac{2 a}{\pi}} \cdot \frac{1}{\sqrt{t} \cdot(t-\tau)^{\frac{1}{4}}} \cdot\left(\frac{t^{2}}{4 a^{2}(t-\tau)}\right)^{\frac{1}{4}} \cdot \exp \left\{-\frac{t^{2}}{4 a^{2}(t-\tau)}\right\}= \\
=\frac{1}{\sqrt{\pi}} \cdot \frac{1}{\sqrt{t-\tau}} \cdot \exp \left\{-\frac{t^{2}}{4 a^{2}(t-\tau)}\right\} .
\end{gathered}
$$

For the second summand we have

$$
\begin{gathered}
\lim _{\nu \rightarrow \frac{1}{2}} K_{\nu}^{(2)}(t, \tau)=\frac{\Gamma\left(-\frac{1}{2}\right)}{\Gamma(+0)} \cdot \sqrt{\frac{2 a}{\pi}} \cdot \frac{\sqrt{\pi}}{2} \cdot \frac{1}{\sqrt{t} \cdot(t-\tau)^{\frac{1}{4}}} \cdot\left(\frac{t^{2}}{4 a^{2}(t-\tau)}\right)^{\frac{1}{4}} \times \\
\quad \times \exp \left\{-\frac{t^{2}}{4 a^{2}(t-\tau)}\right\} \cdot \int_{0}^{\frac{t}{2 a \sqrt{t-\tau}}} \exp \left\{\xi^{2}\right\} d \xi= \\
=\frac{\Gamma\left(-\frac{1}{2}\right)}{2 \cdot \Gamma(+0)} \cdot \frac{1}{\sqrt{t-\tau}} \cdot \exp \left\{-\frac{t^{2}}{4 a^{2}(t-\tau)}\right\} \cdot \int_{0}^{\frac{t}{2 a \sqrt{t-\tau}}} \exp \left\{\xi^{2}\right\} d \xi= \\
=\frac{\Gamma\left(-\frac{1}{2}\right)}{2 \cdot \Gamma(+0)} \cdot \frac{1}{\sqrt{t-\tau}} \cdot \int_{0}^{1} \frac{t}{2 a \sqrt{t-\tau}} \cdot \exp \left\{-\frac{t}{4 a^{2}(t-\tau)} \cdot\left(1-z^{2}\right)\right\} d z= \\
=\frac{\Gamma\left(-\frac{1}{2}\right)}{2 \cdot \Gamma(+0)} \cdot \frac{1}{\sqrt{t-\tau}} \cdot \int_{0}^{1} \frac{t \cdot \sqrt{1-z^{2}}}{2 a \sqrt{t-\tau}} \cdot \exp \left\{-\frac{t^{2}}{4 a^{2}(t-\tau)} \cdot\left(1-z^{2}\right)\right\} \frac{d z}{\sqrt{1-z^{2}}}=0
\end{gathered}
$$

Thus

$$
\lim _{\nu \rightarrow \frac{1}{2}} K_{\nu}(t, \tau)==\frac{1}{\sqrt{t-\tau}} \cdot\left\{\frac{1}{\sqrt{\pi}} \cdot \exp \left\{-\frac{t^{2}}{4 a^{2}(t-\tau)}\right\}-\frac{1}{\sqrt{\pi}} \cdot \frac{1}{\sqrt{t-\tau}}\right\} .
$$

This result can be obtained if we take directly from both sides of (5) the derivative of order $\frac{1}{2}$ :

$$
{ }_{0} D_{t}^{\frac{1}{2}}\left\{\int_{0}^{t} \operatorname{erf}\left(\frac{x}{2 \sqrt{t-\tau}}\right) \cdot \varphi(\tau) d \tau\right\}=
$$




$$
\begin{aligned}
& =\frac{1}{\sqrt{\pi}} \cdot \frac{\partial}{\partial t} \int_{0}^{t} \frac{1}{\sqrt{t-\tau}}\left\{\int_{0}^{\tau} \operatorname{erf}\left(\frac{x}{2 \sqrt{\tau-\tau_{1}}}\right) \cdot \varphi\left(\tau_{1}\right) d \tau_{1}\right\} d \tau= \\
& =\frac{1}{\sqrt{\pi}} \cdot \frac{\partial}{\partial t} \int_{0}^{t}\left\{\int_{\tau_{1}}^{t} \frac{1}{\sqrt{t-\tau}} \cdot \operatorname{erf}\left(\frac{x}{2 a \sqrt{\tau-\tau_{1}}}\right) d \tau\right\} \varphi\left(\tau_{1}\right) d \tau_{1}=\left\|\begin{array}{c}
t-\tau=\eta \\
\tau=t-\eta
\end{array}\right\|= \\
& =\frac{1}{\sqrt{\pi}} \cdot \frac{\partial}{\partial t} \int_{0}^{t}\left\{\int_{0}^{t-\tau_{1}} \frac{1}{\eta^{\frac{1}{2}}} \cdot \operatorname{erf}\left(\frac{x}{2 a \sqrt{t-\tau_{1}-\eta}}\right) d \eta\right\} \varphi\left(\tau_{1}\right) d \tau_{1}= \\
& =\frac{1}{\sqrt{\pi}} \cdot \int_{0}^{t} \frac{\partial}{\partial t}\left\{\int_{0}^{t-\tau_{1}} \frac{1}{\eta^{\frac{1}{2}}} \cdot \operatorname{erf}\left(\frac{x}{2 a \sqrt{t-\tau_{1}-\eta}}\right) d \eta\right\} \varphi\left(\tau_{1}\right) d \tau_{1}= \\
& =\frac{1}{\sqrt{\pi}} \cdot \int_{0}^{t}\left\{\frac{1}{\sqrt{t-\tau_{1}}} \cdot \operatorname{erf}(\infty)-\int_{0}^{t-\tau_{1}} \frac{1}{\eta^{\frac{1}{2}}} \cdot \frac{2}{\sqrt{\pi}} \cdot \exp \left\{-\frac{x^{2}}{4 a^{2}\left(t-\tau_{1}-\eta\right)}\right\} \times \frac{x}{4 a\left(t-\tau_{1}-\eta\right)^{\frac{3}{2}}} d \eta\right\} \varphi\left(\tau_{1}\right) d \tau_{1}= \\
& =\left\|\begin{array}{l}
\eta=\left(t-\tau_{1}\right) \cdot(1-z) \\
d \eta=-\left(t-\tau_{1}\right) d z
\end{array}\right\|= \\
& =\|[3], 3.471\|= \\
& =\frac{1}{\sqrt{\pi}} \cdot \int_{0}^{t} \frac{1}{\sqrt{t-\tau_{1}}}\left(1-\exp \left\{-\frac{t^{2}}{4 a^{2}\left(t-\tau_{1}\right)}\right\}\right) \varphi\left(\tau_{1}\right) d \tau_{1} .
\end{aligned}
$$

Now we show that the kernel of the integral equation (11) has a weak singularity for $\forall \nu, \quad 0 \leq \nu<1$. To do this, we calculate the integral from the kernel $k_{\nu}(t, \tau)$.

$$
\begin{gathered}
J(t, \nu)=\int_{0}^{t} \sqrt{\frac{2 a}{\pi}} \frac{1}{\sqrt{t} \cdot(t-\tau)^{\nu-\frac{1}{4}}} \exp \left\{-\frac{t^{2}}{8 a^{2}(t-\tau)}\right\} \cdot W_{\nu-\frac{1}{4},-\frac{1}{4}}\left\{\frac{t^{2}}{4 a^{2}(t-\tau)}\right\} d \tau= \\
=\left\|\frac{t^{2}}{4 a^{2}(t-\tau)}=y ; \quad t-\tau=\frac{t^{2}}{4 a^{2}} \cdot \frac{1}{y} ; \quad d \tau=\frac{t^{2}}{4 a^{2}} \cdot \frac{1}{y^{2}} d y\right\|= \\
=\int_{\frac{t}{4 a^{2}}}^{\infty} \sqrt{\frac{2 a}{\pi}} \cdot \frac{(2 a)^{2 \nu-\frac{1}{2}}}{t^{2 \nu-\frac{1}{2}}} \cdot \frac{t^{2}}{(2 a)^{2}} \cdot y^{\nu-\frac{1}{4}} \cdot y^{-2} \exp \left\{-\frac{y}{2}\right\} \cdot W_{\nu-\frac{1}{4},-\frac{1}{4}}(y) d y= \\
=\frac{(2 a)^{2 \nu-2}}{\sqrt{\pi}} \cdot t^{2-2 \nu} \cdot \int_{\frac{t}{4 a^{2}}}^{\infty} y^{\left(\nu-\frac{1}{4}\right)-2} \exp \left\{-\frac{y}{2}\right\} \cdot W_{\nu-\frac{1}{4},-\frac{1}{4}}(y) d y= \\
=\left.\frac{(2 a)^{2 \nu-2}}{\sqrt{\pi}} \cdot t^{2-2 \nu} \cdot y^{\nu-\frac{5}{4}} \cdot \exp \left\{-\frac{y}{2}\right\} \cdot W_{\nu-\frac{5}{4},-\frac{1}{4}}(y)\right|_{\frac{t}{4 a^{2}}} ^{\infty}= \\
=\sqrt{\frac{2 a}{\pi}} \cdot t^{-\nu+\frac{3}{4}} \cdot \exp \left\{-\frac{t}{8 a^{2}}\right\} \cdot W_{\nu-\frac{5}{4},-\frac{1}{4}}\left\{\frac{t}{4 a^{2}}\right\} .
\end{gathered}
$$

Let us study the behavior of functions $W_{\nu-\frac{5}{4},-\frac{1}{4}}(z)$ at $t \rightarrow 0$, for this, we represent the function $W_{\nu-\frac{5}{4},-\frac{1}{4}}(z)$ as follows:

$$
W_{\nu-\frac{5}{4},-\frac{1}{4}}(z)=\frac{\Gamma\left(\frac{1}{2}\right)}{\Gamma(2-\nu)} \cdot \mathrm{M}_{\nu-\frac{5}{4},-\frac{1}{4}}(z)+\frac{\Gamma\left(-\frac{1}{2}\right)}{\Gamma\left(\frac{3}{2}-\nu\right)} \cdot M_{\nu-\frac{5}{4}, \frac{1}{4}}(z)=
$$




$$
\begin{gathered}
=\frac{\Gamma\left(\frac{1}{2}\right)}{\Gamma(2-\nu)} \cdot z^{\frac{1}{4}} \cdot e^{-\frac{z}{2}} \cdot \Phi\left(\frac{3}{2}-\nu, \frac{1}{2} ; z\right)+\frac{\Gamma\left(-\frac{1}{2}\right)}{\Gamma\left(\frac{3}{2}-\nu\right)} z^{\frac{1}{4}} \cdot e^{-\frac{z}{2}} \cdot \Phi\left(2-\nu, \frac{3}{2} ; z\right)= \\
=z^{\frac{1}{4}} \cdot e^{-\frac{z}{2}}\left\{\frac{\Gamma\left(\frac{1}{2}\right)}{\Gamma(2-\nu)} \cdot \Phi\left(\frac{3}{2}-\nu, \frac{1}{2} ; z\right)+\frac{\Gamma\left(-\frac{1}{2}\right)}{\Gamma\left(\frac{3}{2}-\nu\right)} z^{\frac{1}{2}} \cdot \Phi\left(2-\nu, \frac{3}{2} ; z\right)\right\} .
\end{gathered}
$$

From here it follows that

$$
\lim _{t \rightarrow 0} \int_{0}^{t} K_{1}(t, \tau) d \tau=0, \forall \nu, 0 \leq \nu<1 .
$$

This means that the integral equation (11) has a unique solution $\varphi_{\lambda}(t), \forall \lambda \in C, f_{2}(t)$. The same can be shown as follows.

The function $k_{\nu}(t, \tau)$ has a singularity at $\tau \rightarrow t$, we define the order of this singularity by using the asymptotics $W_{\lambda, \mu}(z) \approx z^{\lambda} \cdot \exp \left(-\frac{z}{2}\right)$, при $|z| \rightarrow \infty[3,1026 ;(9.227)]$.

We have for the case $\nu>\frac{1}{2}$

$$
\begin{gathered}
k_{\nu}(t, \tau) \approx \sqrt{\frac{2 a}{\pi}} \cdot \frac{1}{\sqrt{t} \cdot(t-\tau)^{\nu-\frac{1}{4}}} \cdot\left[\frac{t^{2}}{4 a^{2} \cdot(t-\tau)}\right]^{\nu-\frac{1}{4}} \cdot \exp \left\{-\frac{t^{2}}{4 a^{2}(t-\tau)}\right\}= \\
=\frac{1}{(t-\tau)^{\nu}} \cdot\left[\frac{t^{2}}{4 a^{2} \cdot(t-\tau)}\right]^{\nu-\frac{1}{2}} \cdot \exp \left\{-\frac{t^{2}}{4 a^{2}(t-\tau)}\right\} \leq \\
\leq c(\nu) \cdot \frac{1}{(t-\tau)^{\nu}} .
\end{gathered}
$$

Here, we use the estimate

$$
x^{\nu-\frac{1}{2}} \cdot \exp \{-x\}<\left(\nu-\frac{1}{2}\right)^{\nu-\frac{1}{2}} \cdot \exp \left\{-\left(\nu-\frac{1}{2}\right)\right\} .
$$

And when $\nu \leq \frac{1}{2}$ the following inequality is used

$$
\begin{aligned}
& \frac{1}{(t-\tau)^{\nu}} \cdot\left[\frac{t^{2}}{4 a^{2} \cdot(t-\tau)}\right]^{\nu-\frac{1}{2}} \cdot \exp \left\{-\frac{t^{2}}{4 a^{2}(t-\tau)}\right\} \leq \\
\leq & \frac{1}{(t-\tau)^{\nu}} \cdot \frac{t^{2 \nu-1}}{(2 a)^{2 \nu-1} \cdot(t-\tau)^{\nu-\frac{1}{2}}}= \\
= & \frac{1}{(2 a)^{2 \nu-1}} \cdot\left(\frac{t-\tau}{t}\right)^{1-2 \nu} \cdot \frac{1}{\sqrt{t-\tau}} \leq(2 a)^{1-2 \nu} \cdot \frac{1}{\sqrt{t-\tau}} .
\end{aligned}
$$

Now, according to (4), we write the solution to problem (1) - (2) in the form

$$
\begin{gathered}
u(x, t)=-\lambda \int_{0}^{t} \operatorname{erf}\left\{\frac{t}{2 a \sqrt{t-\tau}}\right\} \cdot \varphi_{\lambda}(\tau) d \tau+ \\
+\int_{0}^{t} \int_{0}^{\infty} G(x, \xi, t-\tau) \cdot f(\xi, \tau) d \xi d \tau .
\end{gathered}
$$

Thus, it is shown that when $0 \leq \nu<1$ for $\forall \lambda \in C$ the loaded term in (1) - (2) is a weak perturbation.

Thus, the following theorem is valid

Theorem. The boundary value problem (1)-(2) $\forall \nu, 0 \leq \nu<1, \forall \lambda \in C$ has a unique solution $u_{\lambda} \in U$, where

$$
U=\left\{u\left|(x+\sqrt{t})^{-1} \cdot u ; u_{t}-u_{x x} \in M(Q),\left[{ }_{0} D_{t}^{\nu} u(x, t)\right]\right|_{x=t} \in M\left(R_{+}\right)\right\} .
$$




\title{
References
}

1 Дженалиев М.Т. Нагруженные уравнения как возмущения дифференциальных уравнений / М.Т.Дженалиев, М.И.Рамазанов. - Алматы: Ғылым, 2010. - 334 с.

2 Нахушев А.М. Краевые задачи для нагруженных параболических уравнений и их применение к прогнозу уровня грунтовых вод / А.М.Нахушев, В.Н.Борисов // Дифференциальные уравнения. 1977. - Т. 13. - № 1. - C. 105-110.

3 Gradshteyn I.S. Table of Integrals, Series and Products / I.S.Gradshteyn, I.M.Ryzhik. - New York: Academic Press is an imprint of Elsevier, 2007. - P. 1171.

4 Прудников А.П. Интегралы и ряды. - Т. 3. Специальные функции. Дополнительные главы / А.П.Прудников, Ю.А.Брычков, О.И.Маричев. - 2-е изд., испр. - М.: Физматлит, 2003. - 688 с.

\section{М.Т. Жиеналиев, С.А. Искаков, М.И. Рамазанов, Ж.М. Төлеутаева \\ Жүктелген жылуөткізгіштік теңдеуі үшін бірінші шеттік есептің шешімділігі туралы}

\begin{abstract}
Мақалада жазықтықтың бір ширегінде жүктелген жылуөткізгіштік теңдеуі үшін бірінші шеттік есебі қарастырылған. Жүктелген қосылғыш $-x=t$ сызығында уақыттық айнымалысы бойынша $\nu$-ші ретті бөлшек туындының ізі, $0 \leq \nu<1$. Егер $0 \leq \nu<1$ және $\forall \lambda \in \mathrm{C}$ болса, онда жүктеменің әлсіз ауытқу екені көрсетілген, яғни, зерттелетін есептің шенелген функциялар класында жалғыз шешімі бар.

Kiлm сөздер: жүктелген жылуөткізгіштік теңдеу, шеттік есеп, бөлшек туынды, Вольтерраның интегралдық теңдеуі.
\end{abstract}

\section{М.Т. Дженалиев, С.А. Искаков, М.И. Рамазанов, Ж.М. Тулеутаева \\ О разрешимости первой краевой задачи для нагруженного уравнения теплопроводности}

В статье рассмотрена первая краевая задача для нагруженного уравнения теплопроводности в четверти плоскости. Нагруженное слагаемое - след дробной производной порядка $\nu, \quad 0 \leq \nu \leq 1$, по временной переменной на линии $x=t$. Показано, что при $0 \leq \nu \leq 1$ и $\forall \lambda \in \mathrm{C}$ нагрузка является слабым возмущением, то есть исследуемая задача имеет единственное решение в классе ограниченных функций.

Ключевые слова: нагруженное уравнение теплопроводности, краевая задача, дробная производная, интегральное уравнение Вольтерра.

\section{References}

1 Jenaliyev, M.T. \& Ramazanov, M.I. (2010). Nahruzhennye uravneniia kak vozmushcheniia differentsialnykh uravnenii [The loaded equations as perturbations of differential equations]. Almaty: Hylym [in Russian].

2 Nakhushev, A.M. \& Borisov, V.N. (1977). Kraevye zadachi dlia nahruzhennykh parabolicheskikh uravnenii i ikh primenenie k prohnozu urovnia hruntovykh vod [Boundary value problems for loaded parabolic equations and their application to forecast of groundwater level]. Differentsialnye uravneniia - Differential equations, Vol. 13, 1, 105-110 [in Russian].

3 Gradshteyn, I.S. \& Ryzhik, I.M. (2007). Table of Integrals, Series, and Products. New York: Academic Press is an imprint of Elsevier.

4 Prudnikov, A.P., Brychkov, Yu.A. \& Marichev, O.I. (2003). Intehraly i riady. Spetsialnye funktsii. Dopolnitelnye hlavy [Integrals and Series. Special Functions. Additional chapters]. Vol. 3. (2d ed.). Moscow: Fizmatlit [in Russian]. 